\title{
Multi-channel Dual Clocks three-dimensional probability Random Multiple Access protocol for Wireless Public Bus Networks based on RTS/CTS mechanism
}

\author{
Sheng Jie Zhou ${ }^{1}$, Hong Wei Ding ${ }^{1, a}$, Meng Yao Wang ${ }^{1}$, Qian Lin Liu ${ }^{1}$ and Zhi Jun Yang \\ ${ }^{1}$ School of Informaton, Yunnan University, Kunming, 650091, China.
}

\begin{abstract}
A MAC protocol for public bus networks, called Bus MAC protocol, designed to provide high quality Internet service for bus passengers. The paper proposed a multi-channel dual clocks three-demission probability random multiple access protocol based on RTS/CTS mechanism, decreasing collisions caused by multiple access from multiple passengers. Use the RTS/CTS mechanism increases the reliability and stability of the system, reducing the collision possibility of the information packets to a certain extent, improves the channel utilization; use the multichannel mechanism, not only enables the channel load balancing, but also solves the problem of the hidden terminal and exposed terminal. Use the dual clocks mechanism, reducing the system idle time. At last, the different selection of the three-dimensional probabilities can make the system throughput adapt to the network load which could realize the maximum of the system throughput.
\end{abstract}

\section{Introduction}

Intelligent Transportation Systems (Intelligent Traffic System, referred ITS), is the advanced science and technology (information technology, computer technology, data communication technology, sensor technology, electronic control technology, automatic control theory, operations research, artificial intelligence, etc.) to effectively apply the integrated transport, service control and vehicle manufacturing, to strengthen ties vehicles, roads, among users, creating a safety, improve efficiency, improve the environment, energy conservation integrated transport system [1].

With the development of intelligent transportation systems technology, intelligent transportation system will be more widely used in the transportation industry. Therefore, Internet access by bus achieves growing demand. In major cities, every day about the millions of people travel by bus and often takes several minutes or even one hour in traffic.

Compared to other networks, providing Internet services have its obvious channel for the bus access features. Buses usually have more than one passenger. These passengers as a group of mobile node moves and each passenger simultaneously produce different types of Internet service request, for example, news browsing, email access and streaming media data services [2].

Combined bus communication characteristics, the paper proposed a multi-channel dual clocks threedemission probability random multiple access protocol based on RTS/CTS mechanism.
Before the site first sends a request to send a packet transmission frame RTS (Request to Send) to the target site, waiting for the response from the target site CTS (Clear to Send) frame after, it began to transfer packets. If the transmitting station does not receive CTS, then perform a binary exponential backoff algorithm delay retransmission RTS. In this process, we listen to the other node RTS to be delayed for some time to send, in order to ensure the sending node capable of successfully received CTS. Use RTS/CTS handshake (handshake) mechanism, it can ensure that the next transmission packet, no collision, since the RTS/CTS frame while very small, so that the invalid transmission overhead becomes small [3].

Above is the RTS/CTS mechanism. Based on the three-dimensional probability random multiple access protocol, we resolve the "hidden terminal" and "exposed terminal" problem, decreasing collisions caused by multiple access from multiple passengers [4].

Also to reduce the system idle time, we adopt the dual clocks mechanism. When the system is idle, we use continue-time control strategy; when the system is busy, we use the discrete-time control strategy [5].

Last to achieve the multiple business access and improve the system throughput, we join the multi-channel mechanism realization the channel load balancing, meet the different priorities business with different QoS requirements by taking advantage of the priority control of multi-channel multi-service [6].

\section{The analysis of the proposed protocol}

\footnotetext{
a Corresponding author:dhw1964@163.com
} 


\subsection{The analysis of RTS/CTS mechanism}

The channel model of three-dimensional random multiple access protocol is shown in Figure 1.

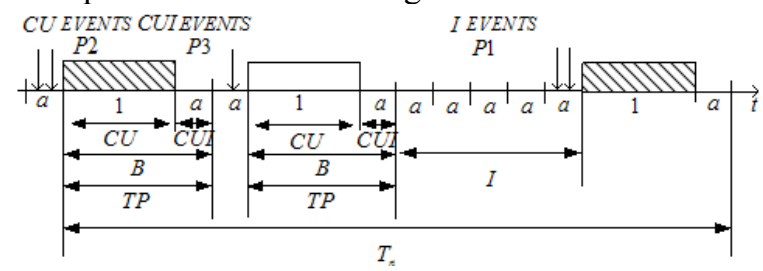

Figure 1. The channel model of three-dimensional random multiple access protocol

In this model, there will be three random events:

- Event that information packets are sent successfully (U events).

- Event that information packets collide with each other (the collision appears, $\mathrm{C}$ events).

- Event that there are no information packets in the channel arrive, the channel is idle (I events) [7].

These three events are forced into: the channel is idle (I events) event, the channel is busy (CU events) and the channel is idle following the CU events (CUI events); the packet is sent successfully or unsuccessfully (combined C events with $\mathrm{U}$ event, denoted by $\mathrm{CU}$ event); force the $\mathrm{CU}$ events and the CUI events into B events [8].

According to the new protocol, if the channel is idle, then the user decides to send an information packet probability $P_{1}$; if the channel is in transmission time, the user listens to the channel with probability $P_{2}$ and $P_{3}$ repectively in time 1 and $a$. This control strategy, $P_{1}, P_{2}$ and $P_{3}$ by three-dimensional selection enables the system utilization and throughput is guaranteed under different load.

The channel model of three-dimensional random multiple access protocol based on RTS/CTS mechanism is shown in Figure 2.

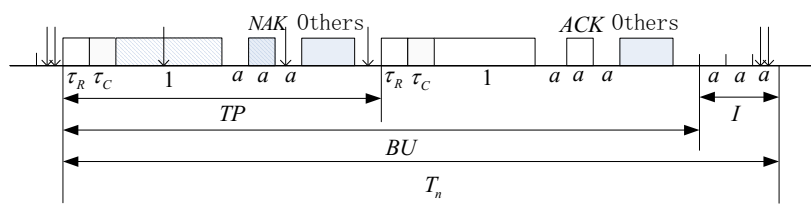

Figure 2 The channel model of three-dimensional random multiple access protocol based on RTS/CTS mechanism

The transmission period is divided into the following sections under the control of the protocol: an interrogation signal RTS, the response signal CTS, an information packet transmission time 1 , ACK monitoring signal $a$, other information content: $\frac{9}{23}\left(1+3 a+\tau_{R}+\tau_{C}\right)$, and the delay time $a$.

When there are $\mathrm{n}$ information packets arriving, the process of channel satisfying the Poisson process, the probability is:

$$
P(n)=\frac{(a G)^{n} e^{-a G}}{n !}
$$

In I events, at idle time slot $a$, if there is no information packets to be sent, its possibility is:

$$
q_{1}^{0}=e^{-a p_{1} G}
$$

In I events, at idle time slot $a$, if there is only one information packet to be sent, its possibility is:

$$
q_{1}^{1}=a p_{1} G e^{-a p_{1} G}
$$

At the transmission period, if there is no information packets to be sent, its possibility is:

$$
q_{2}^{0}=e^{-\left\{p_{2}+\left[\frac{32}{23}\left(3 a+\tau_{R}+\tau_{C}\right)+\frac{9}{23}\right] p_{3}\right\} G}
$$

In the transmission period, if there is only one information packet to be sent, its possibility is:

$$
\begin{gathered}
q_{2}^{1}=\left\{p_{2}+\left[\frac{32}{23}\left(3 a+\tau_{R}+\tau_{C}\right)+\frac{9}{23}\right] p_{3}\right\} \\
G e^{-\left\{p_{2}+\left[\frac{32}{23}\left(3 a+\tau_{R}+\tau_{C}\right)+\frac{9}{23}\right] p_{3}\right\} G}
\end{gathered}
$$

In a cycle, the possibility of continuous $i$ idle events is:

$$
P\left(N_{I}=i\right)=\left(e^{-a p_{1} G}\right)^{i-1} e^{-\left\{p_{2}+\left[\frac{32}{23}\left(3 a+\tau_{R}+\tau_{C}\right)+\frac{9}{23}\right] p_{3}\right\} G}
$$

In a cycle, the possibility of continuous $j \mathrm{~B}$ events is:

$$
P\left(N_{B}=j\right)=\left(1-e^{-a p_{1} G}\right)\left(1-e^{-\left\{p_{2}+\left[\frac{32}{23}\left(3 a+\tau_{R}+\tau_{C}\right)+\frac{9}{23}\right] p_{3}\right\} G}\right)^{j-1}
$$

In a cycle, the possibility of continuous $i$ I events and $j$ B eventsis:

$$
\begin{aligned}
P\left(N_{I}=\right. & \left.i, N_{B}=j\right)=\left(e^{-a p_{1} G}\right)^{i-1}\left(1-e^{-a p_{1} G}\right) \\
& \left(1-e^{-\left\{p_{2}+\left[\frac{32}{23}\left(3 a+\tau_{R}+\tau_{C}\right)+\frac{9}{23}\right] p_{3}\right\} G}\right)^{j-1} e^{-\left\{p_{2}+\left[\frac{32}{23}\left(3 a+\tau_{R}+\tau_{C}\right)+\frac{9}{23}\right] p_{3}\right\} G}
\end{aligned}
$$

The averages number of $i$ continuous I events in a cycle is:

$$
\begin{aligned}
E\left(N_{I}\right)= & \sum_{i=1}^{\infty} \sum_{j=1}^{\infty} i P\left(N_{I}=i, N_{B}=j\right) \\
= & \sum_{i=1}^{\infty} \sum_{j=1}^{\infty} i\left(e^{-a p_{1} G}\right)^{i-1}\left(1-e^{-a p_{1} G}\right) \\
& \left(1-e^{-\left\{p_{2}+\left[\frac{32}{23}\left(3 a+\tau_{R}+\tau_{C}\right)+\frac{9}{23}\right] p_{3}\right\} G}\right)^{j-1} e^{-\left\{p_{2}+\left[\frac{32}{23}\left(3 a+\tau_{R}+\tau_{C}\right)+\frac{9}{23}\right] p_{3}\right\} G} \\
= & \frac{1}{1-e^{-a p_{1} G}}
\end{aligned}
$$

The average number of $j$ continuous B events in a cycle $T_{\mathrm{n}}$ is:

$$
\begin{aligned}
E\left(N_{B}\right)= & \sum_{i=1}^{\infty} \sum_{j=1}^{\infty} j P\left(N_{I}=i, N_{B}=j\right) \\
= & \sum_{i=1}^{\infty} \sum_{j=1}^{\infty} j\left(e^{-a p_{1} G}\right)^{i-1}\left(1-e^{-a p_{1} G}\right) \\
& =\frac{\left(1-e^{-\left\{p_{2}+\left[\frac{32}{23}\left(3 a+\tau_{R}+\tau_{C}\right)+\frac{9}{23}\right] p_{3}\right\} G}\right)^{j-1} e^{-\left\{p_{2}+\left[\frac{32}{23}\left(3 a+\tau_{R}+\tau_{C}\right)+\frac{9}{23}\right] p_{3}\right\} G}}{1-e^{-\left\{p_{2}+\left[\frac{32}{23}\left(3 a+\tau_{R}+\tau_{C}\right)+\frac{9}{23}\right] p_{3}\right\} G}}
\end{aligned}
$$

To the discrete time three-dimensional probability random multiple access protocol with RTS/CTS mechanism, the information packets are sent successfully in two cases.

Firstly the number of information packet transmitted successfully in I events are:

$$
E\left(N_{U_{1}}\right)=\frac{q_{1}^{1}}{1-q_{1}^{0}}=\frac{a p_{1} G e^{-a p_{1} G}}{1-e^{-a p_{1} G}}
$$

The average length of information packet transmitted successfully in I events is:

$$
E\left(U_{1}\right)=E\left(N_{U_{1}}\right) \times 1=\frac{a p_{1} G e^{-a p_{1} G}}{1-e^{-a p_{1} G}}
$$

Secondly the average length of continuous $K U$ events in the TP time in a cycle is: 


$$
\begin{aligned}
E\left(U_{2}\right)= & \sum_{i=1}^{\infty} \sum_{j=1}^{\infty} \sum_{K=0}^{i-1} K P\left(N_{I}=i, N_{B}=j\right) \times 1 \\
= & \sum_{i=1}^{\infty} \sum_{j=1}^{\infty} \sum_{K=0}^{i-1} K\left(e^{-a p_{1} G}\right)^{i-1}\left(1-e^{-a p_{1} G}\right) \\
& \left(1-e^{-\left\{p_{2}+\left[\frac{32}{23}\left(3 a+\tau_{R}+\tau_{C}\right)+\frac{9}{23}\right] p_{3}\right\} G}\right)^{j-1} e^{-\left\{p_{2}+\left[\frac{32}{23}\left(3 a+\tau_{R}+\tau_{C}\right)+\frac{9}{23}\right] p_{3}\right\} G} \\
= & \left\{p_{2}+\left[\frac{32}{23}\left(3 a+\tau_{R}+\tau_{C}\right)+\frac{9}{23}\right] p_{3}\right\} G
\end{aligned}
$$

In a cycle, the average length of time slot that information packet has been successfully sent is:

$$
\begin{aligned}
E(U) & =E\left(U_{1}\right)+E\left(U_{2}\right) \\
& =\frac{a p_{1} G e^{-a p_{1} G}}{1-e^{-a p_{1} G}}+\left\{p_{2}+\left[\frac{32}{23}\left(3 a+\tau_{R}+\tau_{C}\right)+\frac{9}{23}\right] p_{3}\right\} G
\end{aligned}
$$

The average length of $\mathrm{B}$ event in is

$$
E(B)=E\left(N_{B}\right) \times \frac{32}{23}\left(1+3 a+\tau_{R}+\tau_{C}\right)=\frac{\frac{32}{23}\left(1+3 a+\tau_{R}+\tau_{C}\right)}{1-e^{-\left\{p_{2}+\frac{32}{23}\left(3 a+\tau_{R}+\tau_{C}\right)+\frac{9}{23} p_{3}\right\} G}}
$$

The average length of I event is:

$$
E(I)=E\left(N_{I}\right) \times a=\frac{a}{1-e^{-a p_{1} G}}
$$

The throughput of the three-dimensional probability random multiple access protocol with RTS/CTS mechanism is:

$$
\begin{aligned}
S= & \frac{E(U)}{E(B)+E(I)} \\
= & \frac{\frac{a p_{1} G e^{-a p_{1} G}}{1-e^{-a p_{1} G}}+\left\{p_{2}+\left[\frac{32}{23}\left(3 a+\tau_{R}+\tau_{C}\right)+\frac{9}{23}\right] p_{3}\right\} G}{\frac{\frac{32}{23}\left(1+3 a+\tau_{R}+\tau_{C}\right)}{1-e^{\left.-\left\{p_{2}+\left(\frac{32}{23} 3 a+\tau_{R}+\tau_{C}\right)+\frac{9}{23}\right] p_{3}\right\} G}}+\frac{a}{1-e^{-a p_{1} G}}}
\end{aligned}
$$

\subsection{The analysis of dual clocks mechanism}

When the system is idle, the system uses continuous-time control strategy. And if the system is busy, the system uses discrete-time control strategy.

Since the number of idle slots I within the geometric distribution with the mean: $E(N)=\frac{1}{1-e^{-G p_{1} a}}$, an information packet arrive in a time slot with normalized probability: $p_{I 1}=\frac{G p_{1} a e^{-G p_{1} a}}{1-e^{-G p_{1} a}}$, more than an information packet arrives in a time slot with the normalized probability: $p_{I 2}=\frac{1-G p_{1} a e^{-G p_{1} a}-e^{-G p_{1} a}}{1-e^{-G p_{1} a}}$.

Then we get:

$$
E(I)=\left(\frac{1}{1-e^{-G p_{1} a}}-1\right) a+\frac{G p_{1} a^{2} e^{-G p_{1} a}}{2\left(1-e^{-G p_{1} a}\right)}+\frac{\left(1-G p_{1} a e^{-G p_{1} a}-e^{-G p_{1} a}\right) a}{1-e^{-G p_{1} a}}
$$

The throughput of the dual clocks three-dimensional probability random multiple access protocol with $\mathrm{RTS} / \mathrm{CTS}$ mechanism is:

$$
\begin{aligned}
S= & \frac{E(U)}{E(B)+E(I)} \\
= & \left\{\frac{a p_{1} G e^{-a p_{1} G}}{1-e^{-a p_{1} G}}+\left\{p_{2}+\left[\frac{32}{23}\left(3 a+\tau_{R}+\tau_{C}\right)+\frac{9}{23}\right] p_{3}\right\} G\right\} \\
& /\left[\frac{\frac{32}{23}\left(1+3 a+\tau_{R}+\tau_{C}\right)}{1-e^{\left.-\left\{p_{2}+\frac{32}{23}\left(3 a+\tau_{R}+\tau_{C}\right)+\frac{9}{23}\right] p_{3}\right\} G}+\left(\frac{1}{1-e^{-G p_{1} a}}-1\right) a}\right. \\
& \left.+\frac{G p_{1} a^{2} e^{-G p_{1} a}}{2\left(1-e^{-G p_{1} a}\right)}+\frac{\left(1-G p_{1} a e^{-G p_{1} a}-e^{-G p_{1} a}\right) a}{1-e^{-G p_{1} a}}\right]
\end{aligned}
$$

\subsection{The analysis of multi-channel mechanism}

Assuming that nodes have different service requirement have different priorities, setting $\mathrm{N}$ traffic channels in the system, the node occupies the channel according to their business priorities. Assume that each priority unlimited the number of users, the priority from low to high in order is priority 1 , priority $2 \ldots$ priority $\mathrm{N}$.

Priority 1 occupies the channel 1 , priority 2 occupies channel 1 and channel $2 \ldots$ priority i occupies channel 1 to channel i. The arrival information packets on the channel i subject to the Poisson distribution with arriving rate $G_{i}$, the arrival packets of priority $r$ on the channel $\mathrm{i}$ subject to the Poisson distribution with arriving rate $\lambda_{i}=G_{i} /(N-i+1)$. The multichannel mechanism owning $\mathrm{N}$ channels is showed in Figure 3.

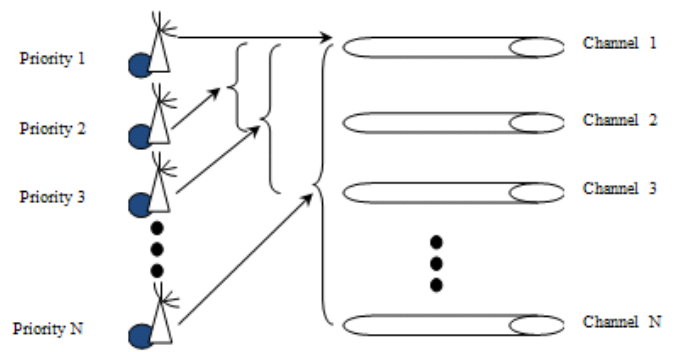

Figure 3 The multichannel mechanism owning $\mathrm{N}$ channels

In the $N$ channels of wireless communication system, because this channel model is a load equilibrium model, so the arrival probabilities of each channel are the same, that is to say:

$$
G_{1}=G_{2}=G_{3}=\cdots=G_{i}=\cdots=G_{N}=G
$$

Basing on the above analysis and computational formula of the systemic throughput, the system total throughput is:

$$
\begin{aligned}
& S^{\prime}=N S \\
& =N\left\{\frac{a p_{1} G e^{-a p_{1} G}}{1-e^{-a p_{1} G}}+\left\{p_{2}+\left[\frac{32}{23}\left(3 a+\tau_{R}+\tau_{C}\right)+\frac{9}{23}\right] p_{3}\right\} G\right\} \\
& /\left[\frac{\frac{32}{23}\left(1+3 a+\tau_{R}+\tau_{C}\right)}{1-e^{-\left\{p_{2}+\left[\frac{32}{23}\left(3 a+\tau_{R}+\tau_{C}\right)+\frac{9}{23}\right] p_{3}\right\} G}}+\left(\frac{1}{1-e^{-G p_{1} a}}-1\right) a\right. \\
& \left.+\frac{G p_{1} a^{2} e^{-G p_{1} a}}{2\left(1-e^{-G p_{1} a}\right)}+\frac{\left(1-G p_{1} a e^{-G p_{1} a}-e^{-G p_{1} a}\right) a}{1-e^{-G p_{1} a}}\right]
\end{aligned}
$$

Then according to the above analysis, we can get the throughput with the priority $l$ :

$$
\begin{aligned}
& S_{p l}=\left(\sum_{i=1}^{l} \frac{1}{N-i+1}\right) S \\
& =\left(\sum_{i=1}^{l} \frac{1}{N-i+1}\right)\left\{\frac{a p_{1} G e^{-a p_{1} G}}{1-e^{-a p_{1} G}}+\left\{p_{2}+\left[\frac{32}{23}\left(3 a+\tau_{R}+\tau_{C}\right)\right.\right.\right. \\
& \left.\left.\left.+\frac{9}{23}\right] p_{3}\right\} G\right\} /\left[\frac{\frac{32}{23}\left(1+3 a+\tau_{R}+\tau_{C}\right)}{1-e^{-\left\{p_{2}+\left[\frac{32}{23}\left(3 a+\tau_{R}+\tau_{C}\right)+\frac{9}{23}\right] p_{3}\right\} G}}+\left(\frac{1}{1-e^{-G p_{1} a}}-1\right) a\right. \\
& \left.+\frac{G p_{1} a^{2} e^{-G p_{1} a}}{2\left(1-e^{-G p_{1} a}\right)}+\frac{\left(1-G p_{1} a e^{-G p_{1} a}-e^{-G p_{1} a}\right) a}{1-e^{-G p_{1} a}}\right]
\end{aligned}
$$

\section{Experimental classification results and analysis}


Based on the above analysis, with the use of simulation tool: MATLAB R2010a, the simulation results are shown as following. During the simulation, transmission delay time: $a=0.1, \tau_{R}=\tau_{C}=0.1$, packet length is: $\frac{32}{23}\left(1+3 a+\tau_{R}+\tau_{C}\right)$.
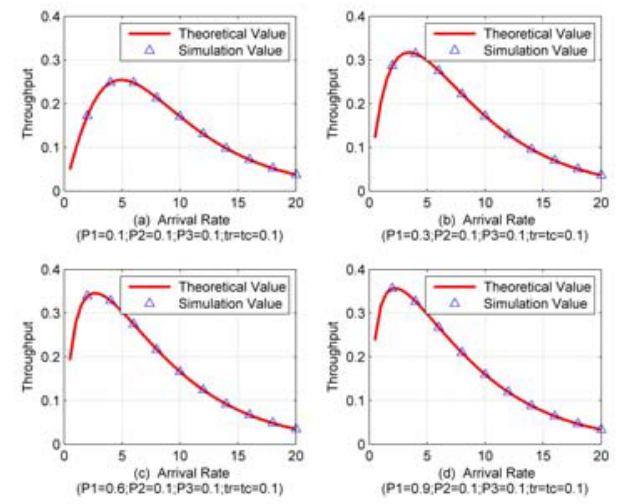

Figure 4 The throughput of the new protocol with different P1.
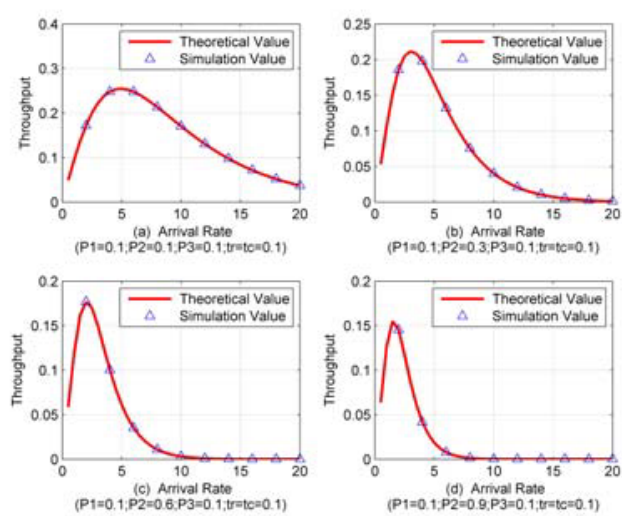

Figure 5 The throughput of the new protocol with different P2.
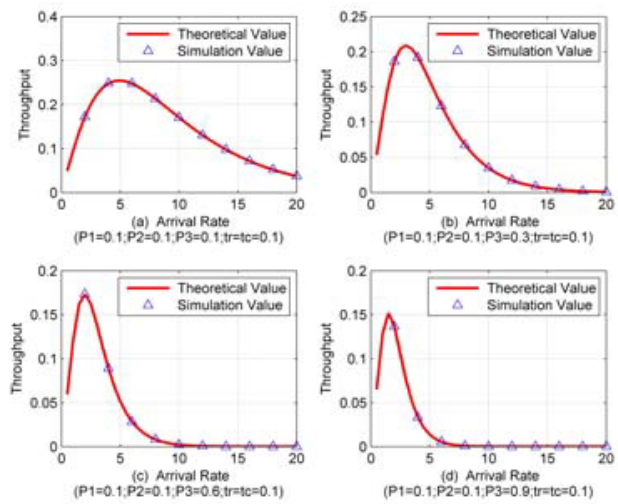

Figure 6 The throughput of the new protocol with different P3.
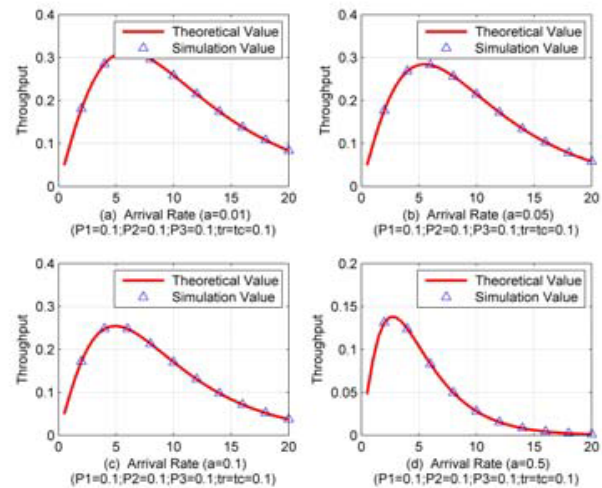

Figure 7 The throughput of the new protocol with different a.
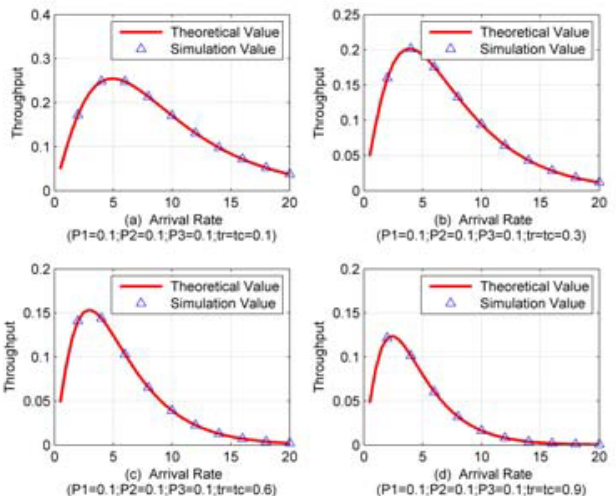

Figure 8 The throughput of new protocol with different tr, tc.

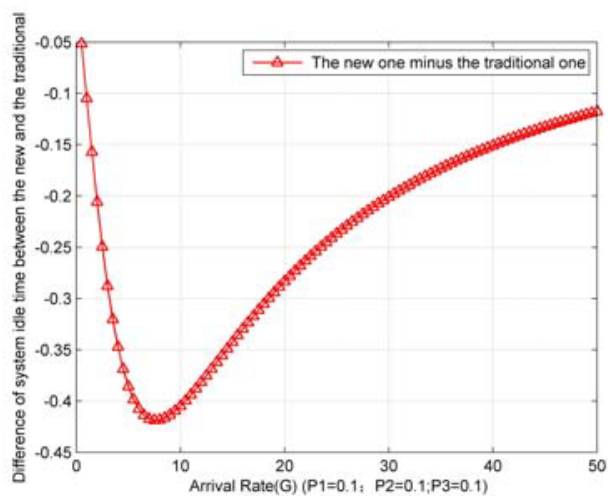

Figure 9 The difference of system idle time between the new protocol and the traditional one.

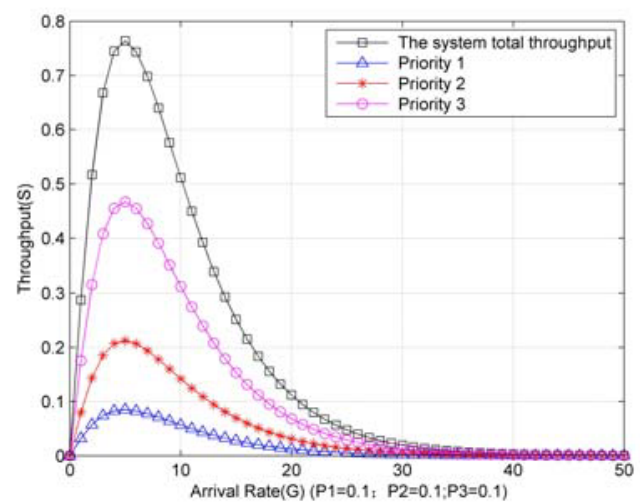


Figure 10 The throughput of the new protocol with 3 channels.

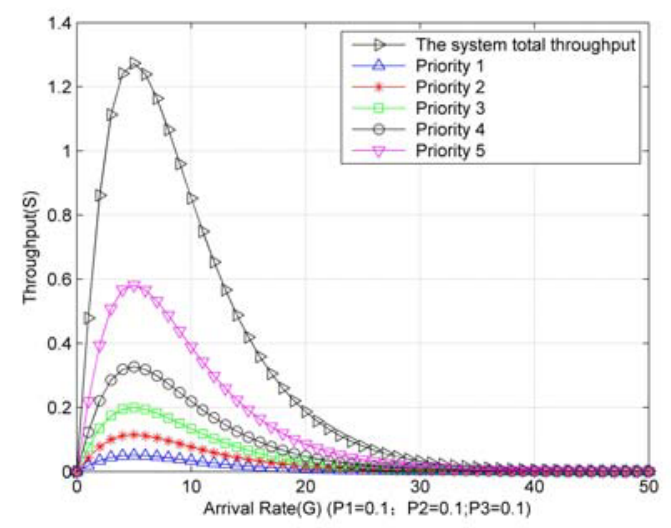

Figure 11 The throughput the new protocol with 5 channels.

Through the above simulation results, we can draw the following conclusions:

1, system simulation coincides with the theoretical value higher degree of consistency proved the theoretical derivation, and proves the accuracy of the simulation.

2, through P1, P2 and P3, we achieve regulation on the system throughput. At low load, P1 increases system throughput can be improved; however, at a high load, P1 increases will reduce the throughput. Because of the low load increases the transmission probability may increase the probability of successfully transmitted packets, and transmit high load increases the probability it will cause more serious conflict. Similarly, when a low load, P2 make a small increase system throughput will increase; a high load, increasing the probability P2 collisions will increase dramatically reduces system throughput. Therefore, we can make the probability of a combination of P1, P2 and P3 at optimum circumstances mentioned in the throughput is greater.

3 , by introducing the dual clock mechanism, it can really reduce the idle rate of the system, especially at low load, idle rate of the system is reduced by a big margin; because at high load, the system is idle rate already high, so the capacity of the mechanism at a high load reducing system idle rate weakened; and in different combinations of probability, idle rate of the system is reduced rates are not the same.

4, and finally through a multi-channel mechanism, not only improves the overall throughput of the system, but also to achieve the different priorities for different bus passengers, providing a different throughput access to multiple services.

\section{Conclusions}

With the development of intelligent transportation systems technology, for providing high quality Internet service in public buses, combined bus communication characteristics, the paper proposed a multi-channel dual clocks three-demission probability random multiple access protocol based on RTS/CTS mechanism. By introduction of the RTS/CTS mechanism increases the reliability and stability of the system, reducing the collision possibility of the information packets to a certain extent, improves the channel utilization; by the use of the multi-channel mechanism, not only enables the channel load balancing, but also solves the problem of the hidden terminal and exposed terminal. By introduction of the dual clocks mechanism, reducing the system idle time. The different selection of the three-dimensional probabilities can make the system throughput adapt to the network load which could realize the maximum of the system throughput. At last, system simulation coincides with the theoretical value higher degree of consistency proved the theoretical derivation, and proves the accuracy of the simulation.

\section{Acknowledgements}

This work was supported by the National Natural Science Foundation of China (61461053, 61461054, 61072079); Natural Science Foundation of Yunnan Province (2010CD023); The Financial Support of Yunnan University (No.XT412004).

\section{References}

1. Shengjie Zhou, Hongwei Ding, Yifan Zhao, Zhijun Yang and Qianlin Liu. Research on the discrete time three-dimensional probability csma protocol in adhoc network [J]. International Journal of Recent Scientific Research, 2015, 6(5): 4257-4262.

2. Xuming. Research and development of mobile Ad Hoc network [J]. Data Communications, 2003 (4): 15-18.

3. Conti M, Giordano S. Mobile ad hoc networking: milestones, challenges, and new research directions [J]. Communications Magazine, IEEE, 2014, 52(1): 85-96.

4. Gandhi C, Arya V. A Survey of Energy-Aware Routing Protocols and Mechanisms for Mobile Ad Hoc Networks [M]. Intelligent Computing, Networking, and Informatics. Springer India, 2014: 111-117.

5. Xiaoping $\mathrm{Wu}$. Research on the hidden and exposed terminal problem in the Ad Hoc network [J]. Microcomputer Information, 2006 (10X): 35-37.

6. Jayasuriya A, Perreau S, Dadej A, et al. Hidden and exposed terminal problem in ad hoc networks[D]. ATNAC 2004.

7. Wang L, Wu K, Hamdi M. Attached-RTS: Eliminating an Exposed Terminal Problem in Wireless Networks [J]. IEEE Transactions on Parallel and Distributed Systems, 2013, 24(7): 12891299.

8. Dongfeng Zhao. Study on A New Method for Continuous-time Systems of Random Access Channel [J]. Journal of Electronics, 1999, 21(1): 3741. 